\title{
Functional disorders of the biliary tract and pancreas
}

\author{
E Corazziari, E A Shaffer, W J Hogan, S Sherman, J Toouli
}

Chair, Committee on Functional Biliary and Pancreatic Disorders, Multinational Working Teams to Develop Diagnostic Criteria for Functional

Gastrointestinal Disorders (Rome II), Cattedra di

Gastroenterologia I, Clinica Medica II, Università "La

Sapienza,"

Rome, Italy

E Corazziari

Co-Chair, Committee on Functional Biliary and Pancreatic

Disorders,

Multinational Working

Teams to Develop

Diagnostic Criteria for

Functional

Gastrointestinal

Disorders (Rome II),

Department of

Medicine,

University of Calgary,

Calgary, Alberta,

Canada

E A Shaffer

Division of

Gastroenterology and

Hepatology,

Milwaukee, WI, USA

W J Hogan

Division of

Gastroenterology and

Hepatology,

Indiana University

School of Medicine,

Indianapolis, IN, USA

S Sherman

Gastrointestinal

Surgical Unit,

Flinders Medical

Centre,

SA, Australia

J Toouli

Correspondence to: Eldon Shaffer, MD, Professor and Head, Department of

Medicine, Foothills Medical Centre, 1403 29th Street

NW, Calgary, Alberta T2N

2T9, Canada. Email: eldon.shaffer@crha-health.ab.ca resonance cholangiopancreatography; Rome II

\section{Abstract}

The term "dysfunction" defines the motor disorders of the gall bladder and the sphincter of Oddi (SO) without note of the potential etiologic factors for the difficulty to differentiate purely functional alterations from subtle structural changes. Dysfunction of the gall bladder and/or SO produces similar patterns of biliopancreatic pain and SO dysfunction may occur in the presence of the gall bladder. The symptom-based diagnostic criteria of gall bladder and SO dysfunction are episodes of severe steady pain located in the epigastrium and right upper abdominal quadrant which last at least 30 minutes. Gall bladder and SO dysfunctions can cause significant clinical symptoms but do not explain many instances of biliopancreatic type of pain. The syndrome of functional abdominal pain should be differentiated from gall bladder and SO dysfunction. In the diagnostic workup, invasive investigations should be performed only in the presence of compelling clinical evidence and after non-invasive testing has yielded negative findings. Gall bladder dysfunction is suspected when laboratory, ultrasonographic, and microscopic bile examination have excluded the presence of gallstones and other structural abnormalities. The finding of decreased gall bladder emptying at cholecystokinin-cholescintigraphy is the only objective characteristic of gall bladder dysfunction. Symptomatic manifestation of SO dysfunction may be accompanied by features of biliary obstruction (biliary-type SO dysfunction) or significant elevation of pancreatic enzymes and pancreatitis (pancreatic-type SO dysfunction). Biliary-type SO dysfunction occurs more frequently in postcholecystectomy patients who are categorized into three types. Types I and II, but not type III, have biochemical and cholangiographic features of biliary obstruction. Pancreatic-type SO dysfunction is less well classified into types. When noninvasive investigations and endoscopic retrograde cholangiopanreatography show no structural abnormality, manometry of both biliary and pancreatic sphincter may be considered.

(Gut 1999;45(Suppl II):II48-II54)

Keywords: biliary tract disease; sphincter of Oddi dysfunction; gallstone disease; pancreatitis; endoscopic retrograde cholangiopancreatography; cholescintigraphy; endoscopic ultrasonography; cholecystokinin; magnetic
Normal gall bladder and sphincter of Oddi function

The gall bladder and the sphincter of Oddi (SO) act as an integrated unit to regulate bile flow from the liver through the biliary tract into the duodenum. ${ }^{1}$ The SO similarly controls pancreatic exocrine output. During fasting, hepatic bile enters the gall bladder for storage. The gall bladder accommodates this increase in volume through receptive relaxation without any significant rise in pressure and by concentrating bile to keep its volume small. The gall bladder evacuates bile by smooth muscle contraction, coordinated with reduced tone in the SO. During fasting, about $25 \%$ of the emptying of gall bladder contents occurs periodically every 100-120 minutes, mediated by motilin, which acts via vagal cholinergic nerves and is synchronized with the migratory motor complex of the intestine. ${ }^{2}$ Eating initiates over $75 \%$ of gall bladder emptying through neural (cephalic and local gastroduodenal reflexes) and hormonal (predominantly cholecystokinin (CCK) acting via cholinergic nerves) influences. ${ }^{3}$ Non-adrenergic, non-cholinergic inhibitory nerves produce $\mathrm{SO}$ relaxation through the release of vasoactive intestinal peptide (VIP) and nitric oxide, acting as postganglionic neurotransmitters. ${ }^{4}$ The neural supply to the biliary tract includes vagal efferent nerves releasing acetylcholine, sympathetic fibers releasing norepinepherine, and sensory nerves containing substance P. Sensory fibers influence the neural response, acting via the vagus through central reflexes. ${ }^{5}$ Derangements of any of these components may lead to intermittent upper abdominal pain, transient elevations of liver or pancreatic enzymes, common bile duct dilatation, or episodes of pancreatitis.

\section{Definition of gall bladder and sphincter} of Oddi dysfunction

Motor dysfunction of the gall bladder and SO has long been suspected as a major feature of several clinical entities that manifest with a similar pattern of upper abdominal pain (table 1). Unlike other functional gastrointestinal disorders, however, abnormalities are beginning to be identified by new technologies, although in many instances a cause-and-effect relationship has not been substantiated. Clinical symptoms may not coincide temporally with the demonstrated abnormality. Further, impaired gall bladder emptying occurs in many patients with cholesterol gallstones, ${ }^{6}$ yet most

Abbreviations used in this paper: $\mathrm{SO}$, sphincter of Oddi; CCK, cholecystokinin; VIP, vasoactive intestinal peptide; ERCP, endoscopic retrograde cholangiopancreatography. 
Table 1 Functional gastrointestinal disorders

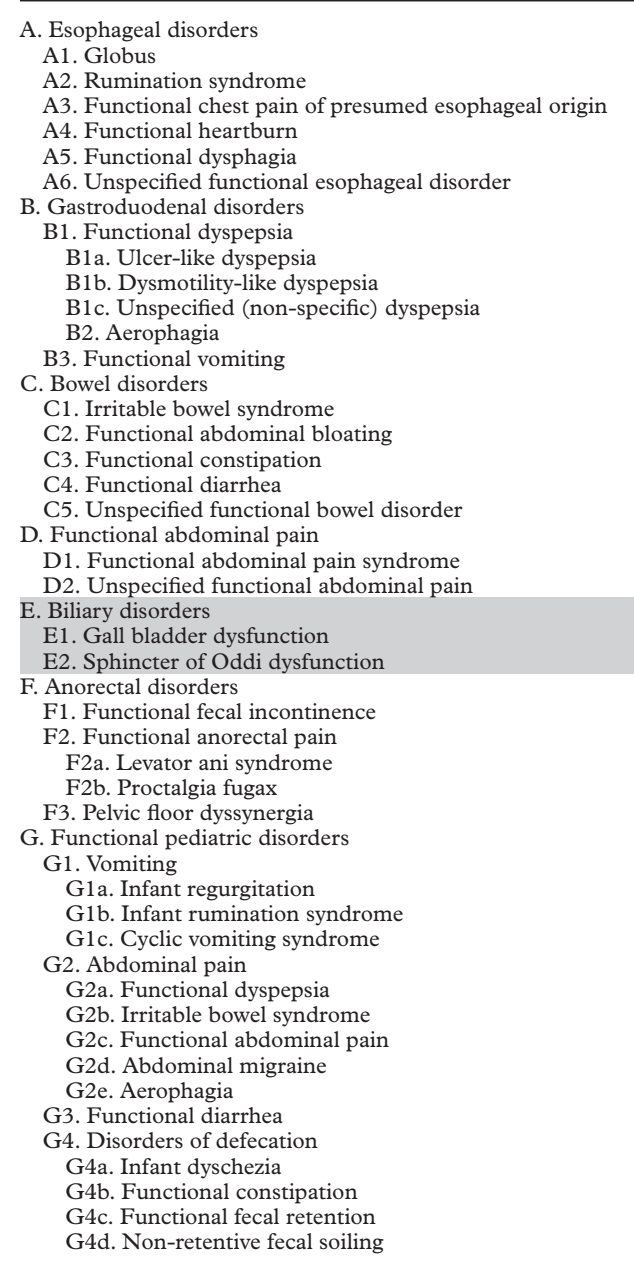

(over $80 \%$ ) never develop symptoms. Structural changes, particularly those indicative of chronic inflammation, may not necessarily correlate with impaired emptying in patients with biliary dyskinesia. ${ }^{7}$ The presumed mechanism for biliary pain is obstruction leading to distension and inflammation. This might result from incoordination between the gall bladder and either the cystic duct or the SO due to increased resistance or tone. Fibrosis and inflammation also can affect the SO, but any clinical relevance is unclear. Rather, for both the gall bladder and $\mathrm{SO}$, motor contraction, sensory afferents, and obstruction/ inflammation all likely play a role in biliarytype pain. Central projections from visceral nociceptors to the thalamus and cortex might lead to a more excitable state with hyperalgesia (severe pain evoked by mildly painful stimuli). Persistent central excitability might then result in allodynia where innocuous stimuli produce pain. ${ }^{8}$ The "hypersensitive biliary tract" may exist, ${ }^{9}$ but the anatomy of the biliary tract precludes ready access to assess this hypothesis scientifically.

Motor dysfunction currently is the most effectively studied of the sources of disorders; it is the only available measure of gall bladder and SO dysfunction. We therefore have adopted the term "dysfunctional disorders of the gall bladder and SO" without attempting to attribute its cause(s) or to identify often subtle morphological changes. This classification consists of:

(1) gall bladder dysfunction, and

(2) SO dysfunction, which may be subdivided into: (a) biliary-type and (b) pancreatictype.

The following clinical observations should be considered with regard to gall bladder and SO dysfunction:

- gall bladder and SO dysfunction manifest symptomatically with the same type of pain;

- although the diagnosis of SO dysfunction is usually made following cholecystectomy, SO dysfunction can manifest clinically in patients with an intact biliary tract;

- psychosocial aspects appear to be variably interrelated with gall bladder and SO dysfunction;

- the syndrome of chronic functional abdominal pain may manifest with clinical characteristics similar to biliopancreatic type of pain.

\section{E1. Gall bladder dysfunction}

The central symptom of gall bladder dysfunction is biliary-type pain. Currently, the only objective characteristic is decreased gall bladder emptying. Available techniques have not clarified its basis (perhaps there is more than one cause) and cannot exclude other entities such as impaired filling or an overly sensitive gall bladder.

EPIDEMIOLOGY

Gallstones are the most common affliction of the gall bladder, but only $10-20 \%$ of patients ever develop symptoms. ${ }^{10}$ Further, there is no association with dyspepsia. ${ }^{11}$ The frequency of biliary pain in those without gallstones may be as high as $7.6 \%$ of men $^{12}$ and $20.7 \%$ of women, ${ }^{13}$ or as low as $2.4 \%$ overall, as reported in an ultrasonographic survey. ${ }^{14}$

DIAGNOSTIC CRITERIA

Episodes of severe steady pain located in the epigastrium and right upper quadrant, and all of the following:

(1) Episodes last 30 minutes or more;

(2) Symptoms have occurred on one or more occasions in the previous 12 months;

(3) The pain is steady and interrupts daily activities or requires consultation with a physician;

(4) There is no evidence of structural abnormalities to explain the symptoms; and

(5) There is abnormal gall bladder functioning with regard to emptying.

The presence of biliary sludge implies gall bladder dysfunction in the form of stasis from impaired emptying but may not necessarily explain the pain. In addition the pain may be associated with one or more of the following: nausea and vomiting; pain radiating to the back and/or right interscapular region; onset after 
meals; and/or awakens the patient at night. In some, these symptoms may be superimposed on a background of low grade chronic abdominal pain of unknown etiology.

\section{RATIONALE FOR CHANGES IN DIAGNOSTIC}

CRITERIA

There are two major changes compared with the Rome I diagnostic criteria. The first refers to the specification of the duration, number of episodes of pain, and the time within which they occur:

(1) 30 minutes has been more firmly established in the literature as the minimum duration of a biliary "colic";

(2) even a single pain episode may be so severe as to justify diagnostic investigation irrespective of the number of episodes, and

(3) the frequency of biliary pain may be so irregular that a time window of only three months has been considered too restrictive.

The second change is point 5 , the specification of the only established functional abnormality.

\section{CLINICAL EVALUATION}

Screening tests

Laboratory-Tests of liver biochemistries and pancreatic enzymes must be normal.

The following tests are necessary to eliminate calculous biliary disease, which can produce similar symptoms.

Ultrasonography-Transabdominal ultrasonography of the upper abdomen is mandatory. The biliary tract and pancreas should be normal and gallstones or sludge absent. Ultrasonography readily detects stones equal to or greater than $3-5 \mathrm{~mm}$ in diameter or biliary sludge within the gall bladder, but it has a low sensitivity for smaller stones or biliary microcrystals. It also has a low yield for stones within the common bile duct. Endoscopic ultrasonography seems to be more sensitive than traditional transabdominal ultrasonography in detecting microlithiasis (tiny stones $<3 \mathrm{~mm}$ ) and sludge within the biliary tract, but the recommendation for its inclusion in standard workups requires further evaluation.

Microscopic bile examination-This procedure is necessary to exclude microlithiasis as a cause. Gall bladder bile can be obtained directly at the time of endoscopic retrograde cholangiopancreatography (ERCP) or by aspiration from the duodenum following stimulation (e.g., CCK-8 $5 \mathrm{ng} / \mathrm{kg}$ i.v. over 10 minutes, or $50 \mathrm{ml} \mathrm{\textrm {MgSO } _ { 4 }}$ instilled into the duodenum). Two types of deposits may be evident: (1) cholesterol microcrystals, which are birefringent and rhomboid shaped, best visualized by polarizing microscopy. ${ }^{15}$ Their presence provides a high diagnostic accuracy for microlithiasis ${ }^{16}$; and (2) bilirubinate granules, which appear as redbrown deposits under conventional light microscopy.

Endoscopy-In the presence of normal laboratory and ultrasonographic findings, endoscopy is usually indicated to exclude upper gastrointestinal diseases.
Tests for gall bladder dysfunction

CCK-cholescintigraphy assessment of gall bladder emptying - This study continuously monitors the hepatic excretion of a radiopharmaceutical into the gall bladder and duodenum, using computer assistance to quantitate changes in radioactivity over the gall bladder. Filling of the gall bladder with radionuclide indicates patency of the cystic duct. Gall bladder emptying is expressed as the gall bladder ejection fraction, the percentage decrease in net gall bladder counts following CCK infusion (CCK-8 slowly infused at $20 \mathrm{ng} / \mathrm{kg}$ over 30 minutes). Reduced emptying, which defines gall bladder dysfunction, can arise from either depressed gall bladder contraction or increased resistance such as elevated tone in the SO. Furthermore, several other conditions that do not necessarily present with biliary colic can be associated with reduced gall bladder emptying. These range from intrinsic gall bladder disease (stones, cholecystitis) to neural and metabolic disorders, drugs, and even the irritable bowel syndrome. Although biliary-type pain is rarely elicited, the test appears to be a marker of this biliary disorder, based on evidence of the beneficial effect of cholecystectomy.

Transabdominal ultrasonography-This test measures gall bladder volume, which if followed serially after a stimulus (meal or CCK), reflects emptying. The technique is operator dependent and the results may not be reproducible in different centers. ${ }^{18}$ Ultrasonographic assessment of gall bladder emptying is currently not the standard for gall bladder dysfunction.

Pain provocation test-Stimulation tests with CCK to duplicate biliary pain have been used historically as a diagnostic investigation. Such tests have low sensitivity and specificity in selecting patients with gall bladder dysfunction who respond to therapy. This may relate to problems in the subjective assessment of pain and the use of bolus injections of CCK, which can induce intestinal contractions.

\section{Diagnostic workup}

Biliary tract symptoms should be evaluated by liver biochemistry, pancreatic enzymes, and ultrasound examination of the abdomen. As a general recommendation we suggest that invasive investigations should be withheld in those patients in whom episodes are infrequent and not accompanied by increased liver function tests.

- If no abnormal findings are detected, CCKcholescintigraphy should be used to assess gall bladder emptying. Abnormal gall bladder emptying ( $<40 \%$ ejection) indicates gall bladder dysfunction.

- If there is no obvious cause for impaired emptying, cholecystectomy is appropriate treatment.

- If gall bladder emptying is normal, bile for microscopic examination to detect cholesterol microcrystals and bilirubinate can be obtained by duodenal drainage, at the time of gastrointestinal endoscopy or during 
ERCP. Magnetic resonance cholangiography or endoscopic ultrasound, where available, can be performed to detect lithiasis.

- If gall bladder emptying is normal, ERCP should be considered. In the absence of common bile duct stones or other abnormalities, SO manometry should be considered if clinically indicated. Evidence of SO dysfunction is an indication for treatment, which may include sphincterotomy.

\section{APPROACH TO TREATMENT}

Medical therapy remains theoretical. It might take the form of: (1) altering gall bladder motor function (e.g., use of motility agents which enhance gall bladder contractility ${ }^{19}$ or ursodeoxycholic acid which worsens motility yet lessens the likelihood of biliary pain ${ }^{20}$ ); (2) reducing visceral hyperalgesia or inflammation (e.g., with non-steroidal anti-inflammatory drugs $\left.{ }^{21}\right)$; or (3) as a last resort, cholecystectomy. CCKcholescintigraphy will identify those patients with impaired emptying. The challenge is to interpret the subgroup that will benefit from treatment. Laparascopic cholecystectomy retains a role in the treatment of gall bladder dysfunction, although favorable outcomes may deteriorate with time, a potential placebo effect of surgery.

\section{E2. Sphincter of Oddi dysfunction}

Sphincter of Oddi dysfunction is the term used to define motility abnormalities of the SO. Because of its strategic position at the duodenal junction of the biliary duct and pancreatic duct, SO dysfunction may result in either biliary or pancreatic disorders. SO dysfunction may be present in patients with an intact biliary tract, but it has been more frequently reported following cholecystectomy.

\section{E2a. Biliary-type SO dysfunction}

Patients present with intermittent episodes of biliary-type pain, sometimes accompanied by biochemical features of transient biliary tract obstruction: elevated serum aminotransferases, alkaline phosphatase, or conjugated bilirubin. These postcholecystectomy patients have been arbitrarily classified according to clinical presentation, laboratory results, and ERCP findings ${ }^{22}$ :

- Patients with biliary-type I SO dysfunction present with pain, elevated liver function tests documented on two or more occasions, delayed contrast drainage, and a dilated common bile duct with a corrected diameter equal to or greater than $12 \mathrm{~mm}$ at ERCP.

- Type II patients present with pain and only one or two of the previously mentioned criteria.

- Type III patients have only recurrent biliary-type pain and none of the above criteria.

The predictability of SO dysfunction varies among these groups, being highest in types I (65-95\%) and II (50-63\%), but less so in type III $(12-28 \%)$. Conversely, the probability that the syndrome of chronic functional abdominal pain (see chapters on Functional bowel disor- ders and Functional abdominal pain) manifests itself as biliary pain is higher in type III patients and less likely in type I.

\section{E2b. Pancreatic-type SO dysfunction}

Sphincter of Oddi dysfunction is less easily classified into types. In its more obvious form (like biliary-type I) pancreatic-type SO dysfunction may present with classic pancreatitis with epigastric pain, which often radiates to the back, and with evidence of elevated serum amylase or lipase. The absence of the traditional causes of pancreatitis (no stones or alcohol misuse) often yields the label of idiopathic recurrent pancreatitis. In a less obvious form (like biliary-type III SO dysfunction), the pain is similar but there is no increase in pancreatic enzymes; in many of these patients the symptomatology may be a manifestation of the syndrome of functional abdominal pain.

\section{EPIDEMIOLOGY}

The prevalence of symptoms suggesting SO dysfunction is about $1.5 \%$ of patients after cholecystectomy, being more frequent in women. ${ }^{23}$ From another perspective, SO dysfunction appears in less than $1 \%$ of patients after cholecystectomy and in $14 \%$ of a selected group of patients complaining of postcholecystectomy symptoms. ${ }^{24}$ In patients with idiopathic recurrent pancreatitis, manometric evidence of SO dysfunction was found to vary between 39 and $90 \% .{ }^{25}{ }^{26} \mathrm{SO}$ dysfunction can involve abnormalities in the biliary sphincter, pancreatic sphincter, or both. The true frequency thus depends on whether one or both sphincters are studied.

DIAGNOSTIC CRITERIA

Episodes of severe steady pain located in the epigastrium and right upper quadrant, and all of the following:

(1) Episodes last 30 minutes or more;

(2) Symptoms have occurred on one or more occasions in the previous 12 months;

(3) The pain is steady and interrupts daily activities or requires consultation with a physician; and

(4) There is no evidence of structural abnormalities to explain the symptoms.

In addition the pain may be associated with one or more of the following:

- The diagnosis is supported by elevated serum aminotransferases, alkaline phosphatase, or conjugated bilirubin, and/or pancreatic enzymes (amylase/lipase).

- Acute recurrent pancreatitis can indicate pancreatic SO dysfunction.

Other clinical features that may be associated with the pain episodes are: nausea and vomiting; pain radiating to the back and/or right interscapular regions (biliary) and/or pain partially alleviated by bending forward (pancreatic); onset after meals; awakens the patient at night.

Sphincter of Oddi dysfunction may exist in the presence of an intact biliary tract with the gall bladder intact. As the symptoms of $\mathrm{SO}$ or 
Table 2 Pressure profile of the sphincter of Oddi measured at the common bile (CBD) and pancreatic $(P D)$ ducts

\begin{tabular}{|c|c|c|c|}
\hline & Normal $^{*} C B D$ & Abnormalt PD & $C B D$ and $P D$ \\
\hline Duct pressure $(\mathrm{mm} \mathrm{Hg})$ & $7.4(1.7)$ & $8.0(1.6)$ & \\
\hline Basal pressure (mm Hg) & $16.2(5.8)(6-25)$ & $17.3(5.8)(8-26)$ & $>40$ \\
\hline \multicolumn{4}{|l|}{ Phasic contractions } \\
\hline Amplitude (mm Hg) & $136.5(25.9)(82-180)$ & $127.5(21.5)(90-160)$ & $>350$ \\
\hline Duration (seconds) & $4.7(0.9)(3-6)$ & $4.8(0.7)(4-6)$ & \\
\hline Frequency (/minute) & $5.7(1.4)(3-10)$ & $5.8(1.5)(3-10)$ & $>7$ \\
\hline \multicolumn{4}{|l|}{ Propagation sequence (\%) } \\
\hline Simultaneous & $55(10-100)$ & $53(10-90)$ & \\
\hline Antegrade & $34(0-70)$ & $35(10-70)$ & \\
\hline Retrograde & $11(0-40)$ & $12(0-40)$ & $>50$ \\
\hline
\end{tabular}

*Values are mean (SD); ranges are given in parentheses. ${ }^{31}$

tAbnormal values for the CBD. ${ }^{32}$

gall bladder dysfunction cannot be readily separated, the diagnosis of SO dysfunction is made commonly following cholecystectomy, or less frequently after proper investigations have excluded gall bladder abnormalities.

RATIONALE FOR CHANGES IN DIAGNOSTIC CRITERIA

There is one major change compared with the Rome I diagnostic criteria. This refers to the specification of the duration, number of episodes of pain, and the time within which they occur:

(1) 30 minutes has been more firmly established in the literature as the minimum duration of a biliary "colic";

(2) even a single pain episode may be so severe as to justify diagnostic investigation irrespective of the number of episodes, and

(3) the frequency of biliary pain may be so irregular that a time window of only three months has been considered too restrictive.

CLINICAL EVALUATION

The only method that can directly assess the motor function of the SO is manometry. This technique is difficult to perform and interpret, is not widely available, and is invasive with potential complications. Because SO dysfunction is relatively uncommon, less invasive procedures should therefore be considered first.

\section{Screening tests}

Liver biochemistry - A transient but significant elevation of liver enzymes and/or bilirubin in close temporal relation to at least two episodes of biliary pain is suspect for SO dysfunction. ${ }^{27}$ Pancreatic enzymes-A significant elevation of either amylase or lipase in close temporal relation to pancreatic pain is suggestive of pancreatitis due to SO dysfunction.

Pain provocative tests-Use of morphine ( \pm prostigmine) historically to detect SO dysfunction was greatly limited by sensitivity and specificity. $^{27}$

Ultrasonographic assessment of duct diameterThe common bile duct is normally $6 \mathrm{~mm}$ or less. ${ }^{28} \mathrm{~A}$ dilated duct may indicate resistance to bile flow through the SO but is not diagnostic as this is evident in $34 \%$ of asymptomatic cholecystectomized subjects. The value of a fatty meal or CCK test to unmask a partially obstructed bile duct has not gained acceptance and neither has secretin stimulation for $\mathrm{SO}$ pancreatic dysfunction.
Choledochoscintigraphy-Following cholecystectomy, $\mathrm{SO}$ tone mainly regulates bile delivery into the duodenum. Dysfunction of the sphincter becomes manifest by a delay in the disappearance of radiopharmaceutical markers of bile from the biliary tract, ${ }^{29}$ or a prolonged transit of radiolabeled bile from the hepatic hilum to the duodenum. ${ }^{30}$ Choledochoscintigraphy is a useful screening method to select patients after cholecystectomy, in whom SO manometry might reveal abnormalities.

\section{Invasive tests}

Endoscopic retrograde cholangiopancreatographyCertain radiologic features at ERCP such as a common bile duct diameter exceeding $12 \mathrm{~mm}$ and delayed emptying of contrast media $(>45$ minutes) suggest SO dysfunction. Additional features are a dilated pancreatic duct $(>5 \mathrm{~mm})$ and delayed emptying of contrast media from the pancreatic duct ( $>10$ minutes). However, variables such as premedication, lack of standardization, and the patient's posture limit their value.

SO manometry-Perendoscopic manometry identifies the sphincter as the zone of elevated resting pressure between the duct (pancreatic or choledochal) and the duodenum. Phasic waves are superimposed (table 2). ${ }^{31}$ Manometric alterations of the SO include: increased basal pressure, increased amplitude of phasic waves, a paradoxical response to CCK, increased frequency of phasic waves, and an increased number of retrograde waves. ${ }^{24-27} 32$ Elevated basal SO pressure is diagnostic of either stenosis or spasm of the sphincter. With sphincteric spasm, SO pressure decreases after administering a smooth muscle relaxant.

\section{Diagnostic workup}

As a general recommendation we suggest that invasive investigations should be withheld in those patients in whom episodes are infrequent and not accompanied by increased liver function tests or pancreatic enzymes. Complications from invasive procedures such as ERCP and SO manometry are more frequent in patients with $\mathrm{SO}$ dysfunction and when performed by inexperienced endoscopists. The following recommendations apply only to skilled endoscopists, preferably in referral centers.

Biliary-type SO dysfunction-

- The evaluation of biliary pain in patients without gall bladders begins with laboratory analyses of liver function and pancreatic enzymes, plus elimination of potential structural causes by: transabdominal ultrasound, analysis of bile for microcrystals, magnetic resonance cholangiography, and endoscopic ultrasound (where available), and ERCP, depending upon the circumstances of the patient and the resources available.

- Choledochoscintigraphy is a useful screening test before SO manometry.

- Patients with type I SO dysfunction may undergo endoscopic sphincterotomy without SO manometry. SO manometry is 
recommended in type II and could be considered in type III dysfunction.

- If SO manometry is normal, look for causes other than SO dysfunction.

- SO stenosis should be treated by endoscopic sphincterotomy.

- In SO dyskinesia, a trial of drug therapy may be in order.

For patients with an intact gall bladder, the workup is part of the same diagnostic algorithm for gall bladder dysfunction.

Pancreatic-type SO dysfunction-

- When ERCP demonstrates no structural abnormality, manometry of both biliary and pancreatic sphincter is indicated.

- Finding biliary SO dysfunction leads to an endoscopic biliary sphincterotomy.

- For pancreatic SO dysfunction alone or with biliary SO dysfunction, standard therapy is operative sphincteroplasty and pancreatic septoplasty. Combined endoscopic biliary and pancreatic sphincterotomy is undergoing investigation.

TREATMENT

The therapeutic approach in patients with SO dysfunction aims to reduce the resistance to the flow of bile or pancreatic juice.

\section{Pharmacotherapy}

Some therapeutic agents have potential but there is limited evidence for their therapeutic usefulness.

- Hormones such as CCK and glucagon can transiently reduce SO tone.

- Calcium channel blockers such as nifedipine at $10-20 \mathrm{mg}$ p.o. decreases the SO pressure and lessens phasic contractions in biliary dyskinesia, benefiting patients with type II SO dysfunction. ${ }^{33}$

- Nitrates decrease sphincteric pressure and can alleviate the symptoms, at least in the short term. ${ }^{34}$

- Botulinum toxin, a potent inhibitor of acetylcholine release, when injected into the sphincter reduces its pressure, improves bile flow, and provides some symptomatic relief. $^{35}$

Such medical therapies have several drawbacks. Calcium channel blockers and nitrates have significant side effects, whereas smooth muscle relaxants are unlikely to be of any benefit in patients with SO stenosis. Responses tend to be transient and long term reports are lacking.

\section{Sphincterotomy}

Endoscopic sphincterotomy is the most widely used therapeutic procedure for patients with biliary-type SO dysfunction, being less expensive and having lower morbidity than transduodenal surgery. Endoscopic sphincterotomy provides symptomatic relief in $55-95 \%$ of patients. The variable outcomes reflect the different criteria used, the methods of data collection (retrospective $v$ prospective), and the techniques used to determine benefit. Pancreatitis in $5-16 \%$ is the most common short term complication of endoscopic sphincterotomy, a rate higher than that for common duct stone extrac- tion. The even higher complication rate with hydrostatic balloon dilatation and placement of biliary (or pancreatic) stents for temporary pain relief does not support their use.

For pancreatic-type SO dysfunction, severance of the pancreatic sphincter, not merely biliary sphincterotomy, may be necessary for a successful outcome. The surgical approach is via transduodenal sphincteroplasty and pancreatic duct septoplasty, yielding $70 \%$ improvement with a low risk of pancreatitis but a high morbidity of $30 \%{ }^{36}$ Endoscopic pancreatic sphincterotomy is another approach under investigation. All such approaches require further study.

\section{Conclusion}

Motility disorders of the gall bladder and SO can cause significant clinical symptoms but are not likely to explain many instances of biliary pain. Clearly, elucidation of the basis for such dysmotility and the detection of a putative hypersensitive biliary tract ${ }^{37}$ should sharpen our diagnostic tools, expand therapeutic options, and benefit those with this disabling problem.

We thank the following reviewers for their critique of the manuscripts and their suggestions: D A Drossman, D Festi, P Portincasa, A Slivka, J Svanvik.

1 Ryan JP. Motility of the gallbladder and biliary tree. In: Johnson LR, ed. Physiology of the gastrointestinal tract. New Johnson LR, ed. Physiology of the gast
York: Raven Press, 1987:695-722.

2 Stolk MF, van Erpecum KJ, Smout AJ, et al. Motor cycles with phase III in antrum are associated with high motilin levels and prolonged gallbladder emptying. Am f Physiol 1993;264:G596-600.

3 Takahashi T, May D, Owyang C. Cholinergic dependence of gallbladder response to cholecystokinin in the guinea pig in vivo. Am f Physiol 1991;261:G565-9.

4 Pauletzki JG, Sharkey KA, Davison J, et al. Involvement of L-arginine-nitric oxide pathways in neural relaxation of the sphincter of Oddi. Eur F Pharmacol 1993;232:263-70.

5 Mawe G. Advances in the neurophysiology of the gallbladder. In: Holle GE, Wood JD, eds. Advances in the innervation of the gastrointestinal tract. Munich: Elsevier Science Publishers, 1992:181-93.

6 Pomeranz IS, Shaffer EA. Abnormal gallbladder emptying in a subgroup of patients with gallstones. Gastroenterology :88:787-91.

7 Westlake PJ, Hershfield NB, Kelly JK, et al. Chronic right upper quadrant pain without gallstones: does HIDA scan predict outcome after cholecystectomy? Am $\mathcal{F}$ Gastroenterol 1990;85:986-90

8 Cervero F, Laird JMA. From acute to chronic pain. Mechanisms and hypothesis. Prog Brain Res 1996;110:3-15.

9 Caroli J. Etude des dyskinesie biliares [thesis]. Toulouse, France: Imprimerie du Sud, 1945.

10 Gracie WA, Ransohoff DF. The natural history of silent gallstones. The innocent gallstones is not a myth: $N$ Engl F Med 1982;307:798-800.

11 Kraagg N, Thijs C, Knipschild P. Dyspepsia-how noisy are gallstones? A meta-analysis of epidemiologic studies of biliary pain dyspeptic symptoms, and food intolerance. Scand f Gastroenterol 1995;30:411-21.

12 GREPCO (The Rome group for epidemiology and prevention of cholelithiasis). The epidemiology of gallstone disease in Rome, Italy. I. Prevalence data in men. Hepatology 1988;8:904-6.

13 GREPCO (Rome group for epidemiology and prevention of cholelithiasis). Prevalence of gallstone disease in an Italian dult female population. Am 7 Epidemiol 1984:119:796805.

14 Barbara L, Sama C, Morselli Labate AM, et al. A population study on the prevalence of gallstone disease: the Sirmione Study. Hepatology 1987;7:913-17.

15 Juniper K, Burson EN. Biliary tract studies II. The significance of biliary crystals. Gastroenterology 1957;32: 175-211.

16 Buscail L, Escourrou J, Delvaux M, et al. Microscopic examination of bile directly collected during endoscopic cannulation of the papilla. Utility in suspected microlithiasis. Dig Dis Sci 1992;37:116-20.

17 Yap L, Wycherley AG, Morphett AD, et al. Acalculous biliary pain. Cholecystectomy alleviates symptoms in patients with abnormal cholescintigraphy. Gastroenterology patients with abnorm

18 Barr RG, Agnesi JN, Schaub CR. Acalcolous gallbladder disease. US evaluation after slow-infusion cholecystokinin stimulation in symptomatic and asymptomatic adults. Radiology 1997;204:105-11. 
$19 \mathrm{Xu}$ QW, Shaffer EA. Cisapride improved gallbladder contractility and bile lipid composition in an animal model Gastroenterology 1993;105:1184-91.

20 Sylwestrowicz TA, Shaffer EA. Gallbladder function during gallstone dissolution. The effect of bile acid therapy in patients with gallstones. Gastroenterology 1988;95:740-8.

21 Thornell E, Svanvik J. Pathophysiological aspects on biliary pain. Scand f Urol Nephrol 1983;75(suppl):45-6.

22 Geenen JE, Hogan WJ, Dodds WJ, et al. The efficacy of endoscopic sphincterotomy after cholecystectomy in patients with sphincter of Oddi dysfunction. $N$ Engl F Med 1989;320:82-7.

23 Drossman DA, Li Z, Andruzzi E, et al. US householder survey of functional GI disorders: prevalence, sociodemography and health impact. Dig Dis Sci 1993;38:1569-80.

24 Bar-Meir S, Halpern Z, Barden E, et al. Frequency of papillary dysfunction among cholecystectomized patients. Hepatology 1984;4:328-30.

25 Geenen JE, Hogan WJ, Dodds WJ. Sphincter of Oddi. In: Sivak MV, ed. Gastroenterological endoscopy. Philadelphia: Sivak MV, ed. Gastroen

26 Sherman S, Troiano FP, Hawes RH, et al. Frequency of abnormal sphincter of Oddi manometry compared with the clinical suspicion of sphincter of Oddi dysfunction. Am $\mathcal{F}$ Gastroenterol 1991;86:586-90.

27 Steinberg WM. Sphincter of Oddi dysfunction. A clinical controversy. Gastroenterology 1988;95:1409-15.

28 Hunt DR Scott AJ. Changes in bile duct diameter after cholecystectomy: 15 year perioperative study. Gastroenterology 1989;97:1485-8.
29 Shaffer EA, Hershfield NB, Logan K, et al. Cholescintigraphic detection of functional obstruction of the sphincter of Oddi. Effect of papillotomy. Gastroenterology 1986;90: 728-33.

30 Corazziari E, Cicala M, Habib FI, et al. Hepatoduodenal bile transit in cholecystectomized subjects. Relationship with sphincter of Oddi function and diagnostic value. Dig Dis Sci 1994;39:1985-93.

31 Guelrud M, Mendoza S. Rossiter G, et al. Sphincter of Oddi manometry in healthy volunteers. Dig Dis Sci 1990;35:3846.

32 Toouli J, Roberts-Thomson IC, Dent J, et al. Manometric disorders in patients with suspected sphincter of Oddi dysfunction. Gastroenterology 1985;88:1243-50.

33 Sand J, Nordback I, Koskinen M, et al. Nifedipine for suspected type II sphincter of Oddi dyskinesia. Am f Gastroenterol 1993;88:530-5.

34 Bar-Meir S, Halpern Z, Bardan E. Nitrate therapy in a patient with papillary dysfunction. Gastroenterology 1983; 78:94-5.

35 Pasricha PJ, Miskovsky EP, Kalloo AN. Intrasphincteric injection of botulinum toxin for suspected sphincter of Oddi dysfunction. Gut 1994;35:1319-21.

36 Toouli J, Di Francesco V, Saccone G, et al. Division of the sphincter of Oddi for treatment of dysfunction associated with recurrent pancreatitis. Br F Surg 1996;83:1205-10.

37 Desaultels SG, Slivka A, Hutson WR, et al. Postcholecystectomy pain syndrome: pathophysiology of abdominal pain in Sphincter of Oddi type III. Gastroenterology 1999;116:9005 .

For further information and updates on Rome II,

visit our website at:

www.romecriteria.org 